\title{
Scheduling the Belgian Soccer League
}

\author{
Dries Goossens and Frits C.R. Spieksma \\ Department of Operations Research, Katholieke Universiteit Leuven \\ Naamsestraat 69, B-3000 Leuven, Belgium \\ dries.goossens@econ.kuleuven.be
}

\section{Introduction}

Especially in Europe, soccer has become big business, involving many parties (e.g. teams, police, broadcasting companies, ...) and a lot of money. Naturally, the schedule of the matches is of great importance, since it has a considerable impact on the costs or revenues of all parties involved. Each party has its (possibly conflicting) constraints and wishes, which makes it hard to generate a schedule that is considered fair and acceptable to all parties. In literature, there are papers on the scheduling of the national soccer league of various countries, e.g., Germany [1], Italy [2], The Netherlands [5] and Austria [1]. However, because of some specific constraints that characterize each of these competitions, the models presented in these papers are not readily applicable to soccer league scheduling problems in other countries. In the next section, we give an overview of the parties involved in scheduling the Belgian Soccer League and their requirements.

\section{Problem description}

The Belgian Soccer League consists of 18 teams, which play a double round robin tournament (i.e., each team plays against each other team twice). The league is intermural, meaning that a team plays in its own stadium or in the stadium of the opponent. Furthermore, for reasons of fairness, the schedule in the second half of the competition should be the same as in the first half with opposite home away pattern (i.e., mirroring). The 34 weekends on which there is a matchday are given by the Royal Belgian Football League (KBVB).

Several other requirements must be taken into account. These requirements are based on conversations we had with a representative of the KBVB. First of all, two teams play in the same stadium and therefore, for each week, if one of these teams plays at home, the other should play an away match and vice versa. The same goes for another pair of teams that share the same advertising panels. Also, a mayor can forbid that a game is played in his or her town on one or more dates. The reason behind this is usually that there is some other event (for instance, a summit with EU leaders) in his town needing the attention of the local police. Furthermore, there are a number of so called "risk matches" for which national police is needed to maintain order. For reasons of security, risk 
games can also be forbidden for given dates and locations. Another requirement is that teams should not play more than two consecutive home (away) matches. Finally, the rights to broadcast live matches are sold to a company that in return requires a calendar with at most one "top match" (i.e., games between two of the four "big" clubs in Belgian) per matchday and at least one top match in the final three matchdays (in order to have a competition that is thrilling until the end).

Apart from these hard constraints, there are quite a number of soft constraints. Matches are normally played on a Saturday, however, the broadcasting company has the right to shift a match to Friday and a match to Sunday on a month's notice. Since this could be problematic for teams that also play a midweek match (e.g. Champions League), matches between those teams should be scheduled as much as possible on weekends that are not preceded or followed by a midweek match. Furthermore, each team has its wishes regarding the calendar. For instance, some teams prefer not to play at home when some other team plays at home, because they are afraid that a part of their spectators would attend the other game. Also, most teams prefer not to play against all top teams consecutively, nor do they like to welcome all top teams at home during the first half of the season. Finally, the number of breaks (i.e. consecutive home (away) matches for a team) should be minimized. The scheduling problem is to decide for each matchday which teams plays against each other and which one of each pair plays at home, satisfying all of the hard constraints and as many soft constraints as possible.

\section{Solution approach}

Currently, this problem is solved by a scheduler of the KBVB who starts from a so-called basic match schedule (BMS). A BMS gives for each team a home-away assignment and the opponent. For instance, if the BMS for team $A$ looks like $-B+E-F+J \ldots$, this means that team $A$ plays away against team $B$ on the first matchday, at home against $E$ on the second, and so on. The BMS that is used is a solution to the double round robin problem with perfect mirroring and minimizes the number of breaks. The teams are then each assigned to a letter in the BMS, taking into account the constraints. The scheduler solves this assignment problem by hand. With a lot of patience and a 30-year experience, this results in a schedule that satisfies most of the hard constraints, but disregards the soft constraints. Not surprisingly, during the last years, a number of teams have expressed their displeasure with the league schedule, with some of them even calling it unbalanced (see [3]).

Obviously, there is room for improvement in assigning teams to the letters from the BMS. We developed a mathematical formulation for this problem, which tries to satisfy the hard constraints (in so far that they are not conflicting). Each of the soft constraints were given a penalty by the KBVB, so that the formula- 
tion minimizes the total penalty of the violated constraints. The model can be solved using Ilog Cplex within the hour and was used to construct the schedule for the season 2006-2007.

Although widening the search to multiple basic match schedules will typically lead to a better calendar, the KBVB has decided to stick with the current BMS in order to get a schedule that is compatible with those of the lower divisions. However, it is our intention to investigate the possibilities of considering other basic match schedules with regard to next year's calendar.

\section{References}

1. Bartsch, T., Drexl, A., Kröger, S.: Scheduling the professional soccer leagues of Austria and Germany. Computers \& Operations Research 33 (2006) 1907-1937

2. Della Croce, F., Oliveri, D.: Scheduling the Italian Football League: an ILP-based approach. Computers \& Operations Research 33 (2006) 1963-1974

3. G.L.R.: Voetbalkalender eerste klasse 2005-2006: Genk boos. De Standaard (2005) June 15 th

4. Nemhauser, G. L., Trick, M.A.: Scheduling a major college basketball conference. Operations Research 46(1998) 1-8

5. Schreuder, J.A.M.: Combinatorial aspects of construction of competition Dutch professional football leagues. Discrete Applied Mathematics 35 (1992) 301-312 